\title{
Ripe Tomato Recognition with Computer Vision
}

\author{
Zhang Fang \\ College of Information, Zhejiang Sci-Tech University, Hangzhou, 310018, China \\ email: zhang_fang2014@163.com
}

Keywords: Computer Vision; Ripe Tomato Recognition; Image Segmentation; Canny Operator

\begin{abstract}
In order to realize the tomato harvest machinery automation, this paper presents a method for automatic recognition of ripe tomato with computer vision. First, the H color component in HSI color space is exploited as the color feature parameter for identification. The RGB tomato image acquired by computer vision is transformed into HIS image, we cut off ripe tomato region based on the gray distribution of $\mathrm{H}$ component using the threshold method. Then, Canny operator is used to edge detection, after corrosion expansion, the tomato centroid coordinates would be marked. Experiments proceed with the image taken by a CCD camera of tomato in different lighting conditions. The results show that, our algorithm can identify ripe tomato under natural light fast and ripe tomatoes in fields can be separated from each other with a very good recognition effect.
\end{abstract}

\section{Introduction}

At present, computer vision technology is more and more widely applied in agricultural automation field [1-2]. Zhou [3] proposed using the information entropy to adaptively determine high, low threshold of Canny operator. Jiang [4] studied the identification and localization of mature tomato binocular stereo vision based method, to obtain the position information of ripe tomato as the greenhouse automation guidance for ripe tomato picking. Xiang [5] proposes a binocular stereo vision measuring methods combining matching method and depth correction model. The ripe tomato recognition based on computer vision is the most critical part of agricultural robot, only the correct recognition of ripe tomato, agricultural machinery talent to be able to accurately picking the ripe tomato. In order to realize the tomato harvesting machinery automation, this paper presents a method for automatic recognition of ripe tomato using computer vision.

\section{Ripe tomato recognition method based on computer vision}

Algorithm of ripe tomato recognition method based on computer vision proposed in this paper steps are as follows:(1) Convert the RGB color space to HSI color space. (2) Mature tomato edge detection based on Canny operator. (3) Marking the two value of each region in the image, and calculate their respective area and axial ratio. (4) To determine whether there are two tomatoes is connected, the phase boundary words, separate the two tomato, centroid or fill in the gap and the calculation of the various tomato, with black dots mark mature tomato centroid.

\section{Mature tomato region segmentation}

In order to better reflect the chroma value difference, 20 RGB images are converted into HIS image. RGB color space is transformed into HIS color space formula is as follows:

$$
\begin{aligned}
& I=\frac{R+G+B}{3} \\
& \left\{\begin{array}{l}
H=W, B \leq G \\
H=2 \pi-W, B<G
\end{array}\right. \\
& S=1-\frac{3 \min (R, G, B)}{R+G+B}
\end{aligned}
$$

Among them, R, G, B are values of 3 primary color (red, green and blue). 


\section{Mature tomato edge detection based on Canny operator}

Such as the use $\mathrm{I}(i, j)$ of image representation, matrix via image Gauss smoothed is $\mathrm{S}(i, j)$, and then calculates the gradient matrix of the image, first get the $\mathrm{x}$ direction and y direction of $\mathrm{S}(i, j)$ partial differential values $\mathrm{P}(i, j)$ and $\mathrm{Q}(i, j)$, the calculation equation[6]:

$$
\begin{aligned}
& P(i, j)=\frac{x}{\sigma^{2}} \exp \left(-\frac{x^{2}+y^{2}}{2 \sigma^{2}}\right) \\
& Q(i, j)=\frac{y}{\sigma^{2}} \exp \left(-\frac{x^{2}+y^{2}}{2 \sigma^{2}}\right)
\end{aligned}
$$

Among them, $\sigma$-Gauss filter convolution kernel width. Amplitude and direction of the image gradient, respectively

$$
\begin{aligned}
& M(i, j)=\sqrt{P^{2}(i, j)+Q^{2}(i, j)} \\
& \theta(i, j)=\arctan (Q(i, j) / P(i, j))
\end{aligned}
$$

\section{Mature tomato centroid labeling}

Mark is a kind of when there are multiple regions in the image, separate them, and describe method respectively. The image plus after the mark, each region has a corresponding marker image, this time as the marked image, and pixel $f(i, j)$ as the marker value, when the region as the background $f(i, j)=0$, when the region as the object of $f(i, j)$ is not 0 mark the same A value, area of the pixel $f(i, j)$ the same value. The moment features of each region were calculated, the formula is

$$
M(p, q)=\sum_{(i, j) \in A} i^{p} j^{q} \quad(p, q=0,1,2, \cdots \cdots)
$$

From the moment of eigenvalue can be calculated characteristic parameters of regional basic, respectively, area, and centroid. Expressed as

Ripe tomato area

$$
S=M(0,0)
$$

Ripe tomato centroid

$$
\left\{\begin{array}{l}
m=M(1,0) / M(0,0) \\
n=M(0,1) / M(0,0)
\end{array}\right.
$$

\section{Experimental results and analysis}

Through the study of image color space conversion, then the $\mathrm{H}$ component of the gray value statistics, find out the mature tomato regional parts, and through a variety of methods of image analysis to remove the noise due to illumination and other factors, the final segmentation of mature tomato area and find the centroid, ripe tomato recognition results such as shown in Fig. 1. Fig. 1 (a) is the original image; Fig. 1 (b) is the final segmentation image (black spot centroid). In order to make the image after segmentation only has mature tomato information, the need for black small region elimination. Because the black small area and really mature tomatoes region is very small compared with the area, so the threshold segmentation method (based on how many pixels as threshold), these black area are removed.

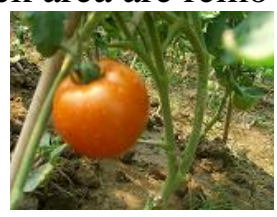

(a)

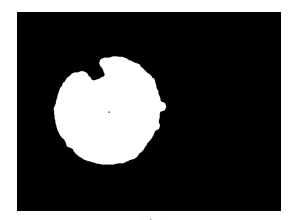

(b)

Fig. 1 the recognition result of ripe tomato 


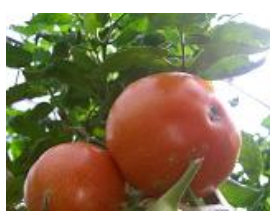

(a)

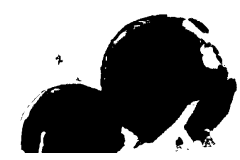

(e)

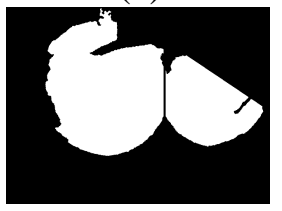

(i)

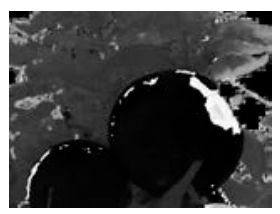

(b)

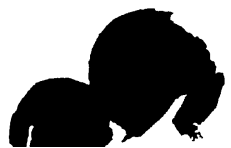

(f)

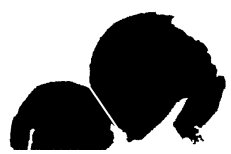

(j)

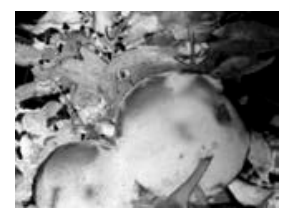

(c)

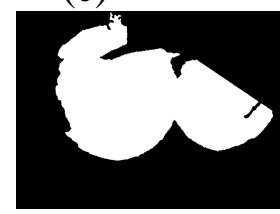

(g)

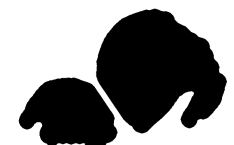

(k)

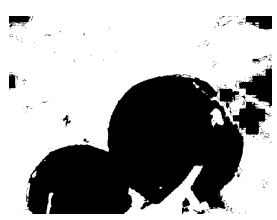

(d)

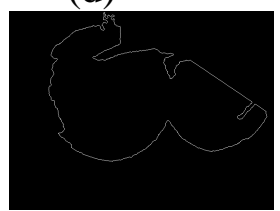

(h)

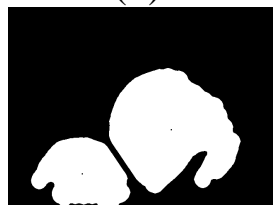

(l)

Fig. 2 overlaps of ripe tomato recognition

How to judge whether the two tomatoes overlap, and separate the two tomatoes is the difficulty of ripe tomato recognition, this algorithm is a good solution to this problem. Fig. 2 is the overlap of ripe tomato recognition results, Fig. 2 (a) is the original image, Fig. 2 (b) is the H image, Fig. 2 (c) is the $\mathrm{S}$ image, Fig. 2 (d) is to partition the image $\mathrm{H}$, figure 2 (e) is to partition the image $\mathrm{S}$, figure 2 (f) is the image after denoising, Fig. 2 (g) is a spin image after the turn, Fig. 2 (h) is the edge detection of image, Fig. 2 (i) is to separate the overlapping image after the ripe tomato, Fig. 2 (j) is to restore the image position, Fig. $2(\mathrm{k})$ is the corrosion image after expansion, Fig. 2 (l) is the image centroid after the mark.

\section{Conclusion}

A method based on computer vision in this paper improved the degree of automation of ripe tomato recognition, provided an important basis for the automation of ripe tomato, tomato automatic harvesting mature automatic classification and mature tomato quality detection. Due to the general agricultural harvest with a common recognition, so this method is not only suitable for mature tomato, also have reference value for the automatic harvesting mature apple, citrus and other agricultural products.

\section{References}

[1] Yamamoto Kyosuke, Yoshioka Yosuke, Ninomiya Seishi. Detection and counting of intact tomato fruits on tree using image analysis and machine learning methods[C]. 5th International Conference, Trends in Agricultural Engineering, 2013: 664-667.

[2] Zhao Jiewen, Liu Muhua, Yang Guobin. Discrimination of mature tomato based on his color space in natural outdoor scenes[J]. Transactions of the Chinese Society of Agricultural Machinery, 2004, 35(5): 122-124.

[3] Zhou Zhiyu, Liu Yingchun, Zhang Jianxin. Orange edge detection based on adaptive Canny operator[J]. Transactions of the Chinese Society of Agricultural Engineering, 2008, 24(3): 21-24.

[4] Jiang Huanyu, Peng Yongshi, Shen Chuan, et al. Recognizing and locating ripe tomatoes based on binocular stereo vision technology[J]. Transactions of the CSAE, 2008,24(8):279-283.

[5] Xiang Rong, Ying Yibin, Jiang Huanyu, et al. Localization of tomatoes based on binocular stereo vision[J]. Transactions of the Chinese Society of Agricultural Engineering (Transactions of the CSAE), 2012, 28(5): 161-167. 
[6] Canny J F. A computational approach to edge detection[J]. IEEE Transactions on Pattern Analysis and Machine Intelligence, 1986, 8 (6): 679-698. 